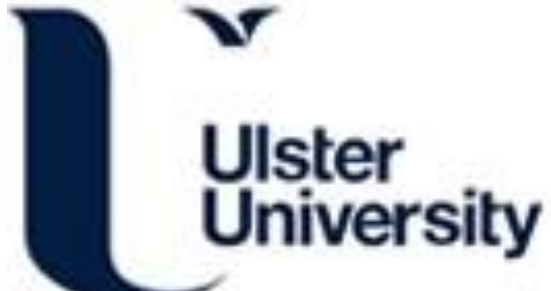

\section{Crystalline Si nanoparticles below crystallization threshold: Effects of collisional heating in non-thermal atmospheric-pressure microplasmas}

Askari, S., Levchenko, I., Ostrikov, K., Maguire, PD., \& Mariotti, D. (2014). Crystalline Si nanoparticles below crystallization threshold: Effects of collisional heating in non-thermal atmospheric-pressure microplasmas. Applied Physics Letters, 104(16). https://doi.org/10.1063/1.4872254

Link to publication record in Ulster University Research Portal

\section{Published in:}

Applied Physics Letters

Publication Status:

Published (in print/issue): 01/01/2014

DOI:

10.1063/1.4872254

\section{Document Version}

Publisher's PDF, also known as Version of record

\section{General rights}

Copyright for the publications made accessible via Ulster University's Research Portal is retained by the author(s) and / or other copyright owners and it is a condition of accessing these publications that users recognise and abide by the legal requirements associated with these rights.

\section{Take down policy}

The Research Portal is Ulster University's institutional repository that provides access to Ulster's research outputs. Every effort has been made to ensure that content in the Research Portal does not infringe any person's rights, or applicable UK laws. If you discover content in the Research Portal that you believe breaches copyright or violates any law, please contact pure-support@ulster.ac.uk. 


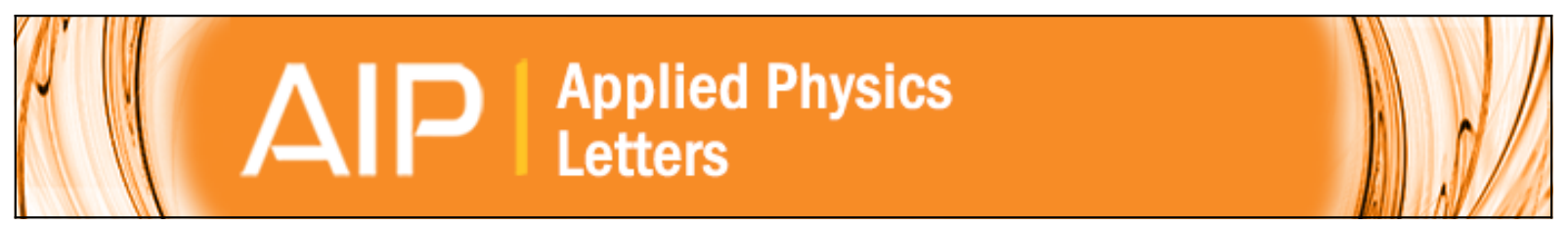

\section{Crystalline Si nanoparticles below crystallization threshold: Effects of collisional heating in non-thermal atmospheric-pressure microplasmas}

S. Askari, I. Levchenko, K. Ostrikov, P. Maguire, and D. Mariotti

Citation: Applied Physics Letters 104, 163103 (2014); doi: 10.1063/1.4872254

View online: http://dx.doi.org/10.1063/1.4872254

View Table of Contents: http://scitation.aip.org/content/aip/journal/apl/104/16?ver=pdfcov

Published by the AIP Publishing

\section{Articles you may be interested in}

Ge nanoparticle formation by thermal treatment of rf-sputtered $\mathrm{ZrO} / \mathrm{ZrGe} 2 \mathrm{O} 3$ superlattices

J. Appl. Phys. 113, 044303 (2013); 10.1063/1.4780033

Dynamical process of $\mathrm{KrF}$ pulsed excimer laser crystallization of ultrathin amorphous silicon films to form $\mathrm{Si}$ nano-dots

J. Appl. Phys. 111, 094320 (2012); 10.1063/1.4716467

Silicon nanoparticle synthesis by short-period thermal anneals at atmospheric pressure in argon

J. Vac. Sci. Technol. A 29, 051508 (2011); 10.1116/1.3620422

Ellipsometry investigation of the amorphous-to-microcrystalline transition in a-Si:H under hydrogen-plasma treatment

J. Appl. Phys. 107, 083509 (2010); 10.1063/1.3393273

Size-dependent crystallization of Si nanoparticles

Appl. Phys. Lett. 88, 093119 (2006); 10.1063/1.2182018

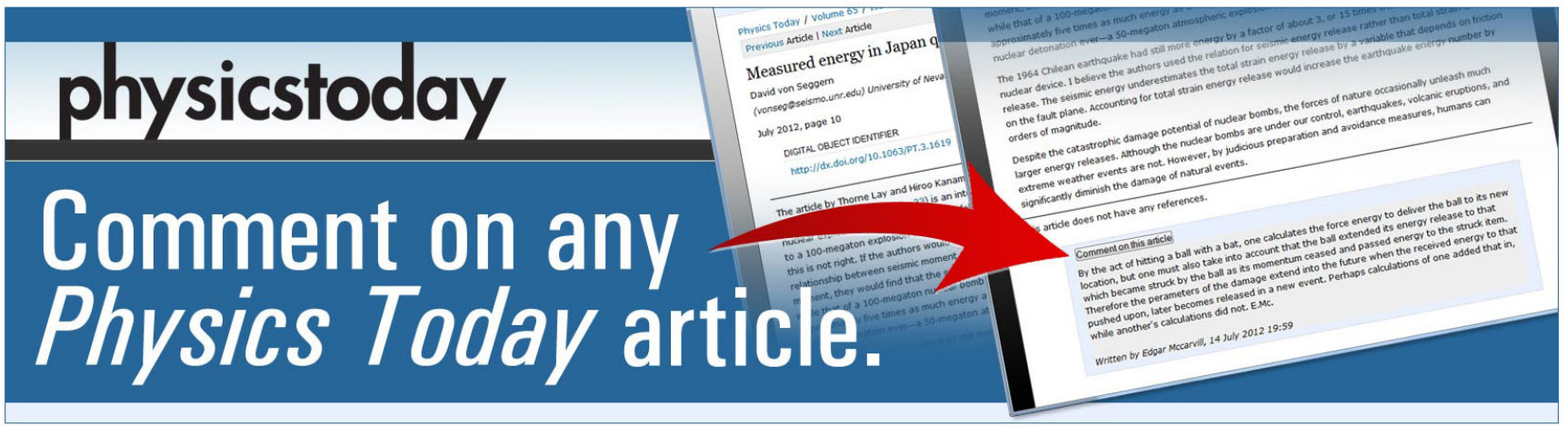




\title{
Crystalline Si nanoparticles below crystallization threshold: Effects of collisional heating in non-thermal atmospheric-pressure microplasmas
}

\author{
S. Askari, ${ }^{1, a)}$ I. Levchenko, ${ }^{2,3}$ K. Ostrikov, ${ }^{2,3,4}$ P. Maguire, ${ }^{1}$ and D. Mariotti ${ }^{1}$ \\ ${ }^{1}$ Nanotechnology and Integrated Bio-Engineering Centre (NIBEC), University of Ulster, \\ Newtownabbey BT37 OQB, United Kingdom \\ ${ }^{2}$ Plasma Nanoscience Centre Australia (PNCA), CSIRO Materials Science and Engineering, P.O. Box 218, \\ Lindfield, NSW 2070, Australia \\ ${ }^{3}$ School of Physics, The University of Sydney, Sydney, NSW 2006, Australia \\ ${ }^{4}$ School of Chemistry, Physics, and Mechanical Engineering, Queensland University of Technology, Brisbane, \\ QLD 4000, Australia
}

(Received 11 March 2014; accepted 4 April 2014; published online 22 April 2014)

\begin{abstract}
Nucleation and growth of highly crystalline silicon nanoparticles in atmospheric-pressure lowtemperature microplasmas at gas temperatures well below the Si crystallization threshold and within a short $(100 \mu \mathrm{s})$ period of time are demonstrated and explained. The modeling reveals that collisionenhanced ion fluxes can effectively increase the heat flux on the nanoparticle surface and this heating is controlled by the ion density. It is shown that nanoparticles can be heated to temperatures above the crystallization threshold. These combined experimental and theoretical results confirm the effective heating and structure control of $\mathrm{Si}$ nanoparticles at atmospheric pressure and low gas temperatures. C 2014 AIP Publishing LLC. [http://dx.doi.org/10.1063/1.4872254]
\end{abstract}

Semiconductor nanoparticles (NPs) are of strong interest for a wide range of applications including photovoltaic cells, ${ }^{1}$ light emitting devices, ${ }^{2}$ fluorescent tags for biomedical applications, ${ }^{3}$ and electronic devices. ${ }^{4}$ Contrary to bulk semiconductors, the bandgap of semiconductor NPs can be controlled by varying their size which affects electron confinement. Among the different semiconductor materials, group IV NPs are of special interest as they are, at least in bulk form, non-toxic, biocompatible and have been widely used on an industrial scale ( $\mathrm{Si}, \mathrm{C}$, and $\mathrm{Ge}$ ). In particular, silicon NPs may offer great opportunities originating from the unique interplay between quantum effects, surface states, and direct/indirect transition dynamics. ${ }^{5-7}$ The crystalline structure of these materials demands high synthesis temperatures that are not generally compatible with conventional wet chemistry. On the other hand, plasma-based processes offer synthesis avenues for a wide range of NPs compositions with some unique advantages (e.g., charging of NPs inside the plasma reduces or even completely prevents the NP agglomeration). ${ }^{8,9}$

Non-thermal atmospheric pressure plasmas (APPs) are highly versatile for the synthesis and processing of nanomaterials, as they can be easily designed and implemented in many different configurations without the need of vacuum systems; these characteristics could potentially facilitate scaling up and integration in industrial processes. The application of APPs for the synthesis of nanomaterials including NPs has been investigated within the last few years, ${ }^{10,11}$ and the synthesis of metal NPs has been often reported. ${ }^{12}$ Other materials such as silicon are less investigated and infrequently reported $^{13-15}$ due to a range of synthesis challenges and difficulties in controlling NPs important properties. For instance, in addition to size-dependent characteristics, the

\footnotetext{
${ }^{\text {a) }}$ Author to whom correspondence should be addressed. Electronic mail: askari-s@email.ulster.ac.uk
}

properties of semiconducting NPs depend strongly on the crystalline/amorphous nature, with dramatic consequences for applications. ${ }^{16,17}$ In this context, heating of NPs plays a fundamental role, in particular, to determine the crystalline versus amorphous structure. Furthermore, optimized plas$\mathrm{ma} /$ growth parameters are required to ensure sufficient heating for the fabrication of highly crystalline NPs; however, heating needs to be carefully controlled to avoid overheating and damage to the crystalline structure by ion bombardment, which could hinder the growth process.

Although heating of NPs in low pressure non-thermal plasmas has been investigated, ${ }^{18-21}$ still many aspects are not fully understood. It is now accepted that the particle temperature in low-pressure plasmas can exceed the gas temperature through ion/electron fluxes reaching the surface of the particle. The study of NP heating in APP presents new aspects that require revisiting the theoretical framework. In APP, intense conduction-based cooling of particle via the surrounding gas can limit the particle temperature rise relative to the gas temperature. On the other hand, high values for electron density $\left(\sim 10^{11} \mathrm{~cm}^{-3}\right.$ to several orders higher $)$ can be reached in cold APP microreactors (also known as microplasmas), ${ }^{10}$ which can lead to intense particle surface heating through surface reactions with charged species.

Here, we present experimental results on the synthesis of narrowly size-distributed crystalline silicon nanoparticles well below the silicon crystallization threshold. An efficient plasma-associated heating resulted in the formation of highly crystalline nanoparticles without any agglomeration. Our work demonstrates that the atmospheric-pressure microplasmas is an efficient tool for the structure control of the plasma-nucleated nanostructures. The plasma parameters were measured using optical emission spectroscopy (OES), and the results were utilized as input parameters to an analytical model that was employed to calculate the NPs electric charge, ion flux; and finally, the nanoparticle temperature. 
It should be noted that our model is applicable to any nanoparticle, irrespective of the material composition.

The schematic of the experimental apparatus considered is shown in Figure 1. The plasma is sustained by supplying radio frequency (RF) power at $13.56 \mathrm{MHz}$ to two copper ring electrodes placed around a quartz capillary with 0.7 internal diameter and $1 \mathrm{~mm}$ external diameter. The flow ratio of argon versus a pre-mixed mixture of argon-silane (50 ppm silane in Ar) is adjusted by regulating two separate mass flow controllers. The plasma microreactor as shown in Figure 1 is housed in a stainless steel chamber to avoid air contamination. The pressure is maintained at $1 \mathrm{~atm}$ while a continuous flow of nitrogen through the chamber is pumped out at a constant flow rate.

For the results presented in this paper, the discharge and gas mixture parameters are fixed at the following conditions: RF power $100 \mathrm{~W}$ (measured at the power supply), $200 \mathrm{sccm}$ argon flow rate, $50 \mathrm{sccm}$ argon/silane flow rate, and $1 \mathrm{~mm}$ electrode spacing. These conditions result in an overall silane concentration of $10 \mathrm{ppm}$. The electrical properties of the discharge are evaluated by an RF voltage-current (MKS VI$\mathrm{RF}$ ) probe placed after the matching unit, at the RF feedthrough outside the chamber.

At the condition described above, the plasma microreactor produces NPs (Figure 2) that are collected downstream directly into a vial with ethanol. NPs are characterized by transmission electron microscopy (TEM) with a JEOL JEM2100F. For TEM analysis, a few drops of the NPs/ethanol colloid are drop-casted on TEM grids. The bright-field TEM images in Figures 2(a) and 2(c) demonstrate that the NPs are spherical with size less than $5 \mathrm{~nm}$ and without any agglomeration. X-ray photoelectron spectroscopy (XPS) and energydispersive X-ray spectroscopy (EDS) confirm the presence of a silicon core with a degree of surface oxidation; XPS and TEM-EDS results can be found in the supplementary material, ${ }^{40}$ which also include photoluminescence and absorption

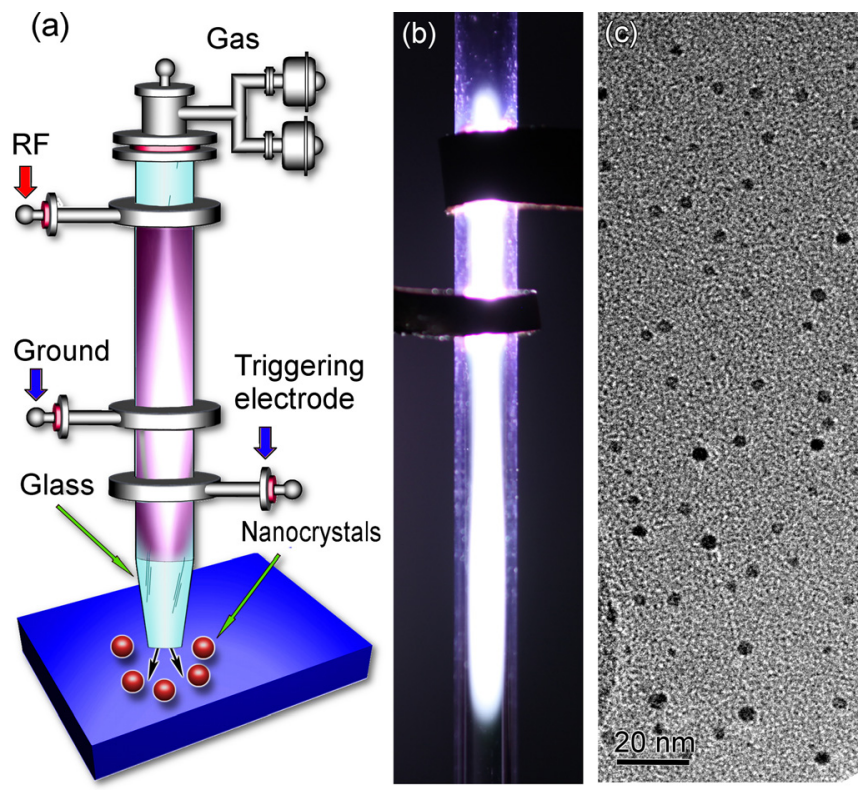

FIG. 1. (a) Schematic of the plasma reactor, (b) photo of the microplasma generated inside a quartz capillary, and (c) representative TEM images of the silicon nanoparticles synthesized in microplasma.
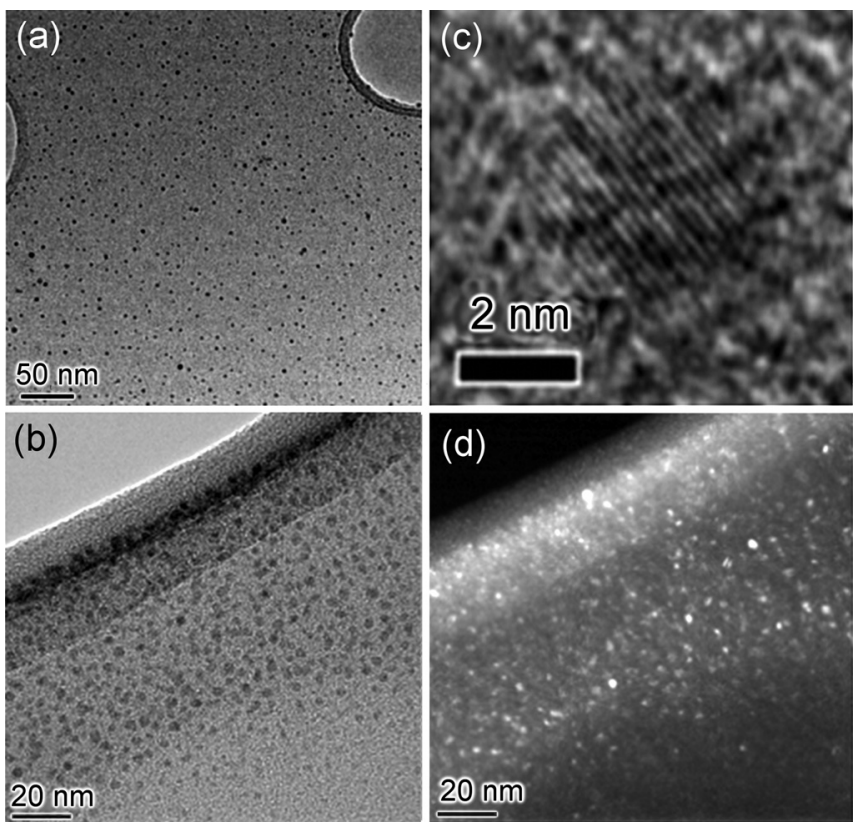

FIG. 2. Transmission electron microscope images of the silicon nanoparticles (a)-(d): the spacing of the fringes in (c) is $0.20 \mathrm{~nm}$ which corresponds to the (220) plane of silicon, and (d) illustrates the dark field image of the crystalline nanoparticles of (b).

measurements of the Si NPs in colloid as well as the size distribution determined from TEM images. Figure 2(c) shows fringes with $0.2 \mathrm{~nm}$ spacing; in light of our XPS and TEMEDS analysis (see supplementary material ${ }^{40}$ ), we have assigned this spacing to that of the (220) plane of silicon. The dark-field TEM image of Figure 2(b) is shown in Figure 2(d); the bright spots in this image confirm the crystallinity of a larger set of NPs.

In order to determine the plasma parameters, OES has been used. OES measurements have been taken for the same plasma conditions as for the synthesis of Si NPs. The light from the plasma is collected through a fiber optic placed $5 \mathrm{~mm}$ away from the plasma and pointing perpendicularly to the mid-point of the quartz capillary tube between the electrodes. The fiber optic is then connected to a spectrograph (Sharmok 3030i) equipped with two different gratings of 1200 grooves $/ \mathrm{mm}$ and 150 grooves $/ \mathrm{mm}$ and a charged coupled device (CCD) detector (Andor iStar). The relation from the rotational levels of $\mathrm{OH}$ radicals is used here to measure the gas temperature, following the previously reported procedures. $^{22,23}$ The method is based on the comparison of synthetic spectra (from LIFBASE software ${ }^{24}$ ) with the measured spectra. The best fit of the simulated profile with the measured intensity reveals the magnitude of the gas temperature, in our case with an estimated error of $\pm 50 \mathrm{~K}$. For measuring the gas temperature, the reactor is operated in open atmosphere as the $\mathrm{OH}$ line is not visible with a sealed chamber.

The electron density is determined from Stark broadening of the $\mathrm{H}_{\beta}(\lambda=486.1 \mathrm{~nm})$ emission line. The Stark broadening of Balmer lines of hydrogen, $\mathrm{H}_{\alpha}, \mathrm{H}_{\beta}$, and $\mathrm{H}_{\gamma}$ has been widely used for evaluating electron density in atmospheric pressure plasmas. ${ }^{22,25,26}$ For the plasma conditions considered here, other effects contributing to the broadening of the emission line are instrumental, Doppler, and Van der Waals 
broadenings. The Stark broadening with a Lorentzian shape is derived by deconvoluting the measured Voigt profile of the line.

The excitation temperature $\left(T_{e x c}\right)$ is calculated from Boltzmann plots for $4 p$ and $5 p$ levels of argon using the corresponding emission lines; $T_{\text {exc }}$ of argon gives an underestimation of the electron temperature in non-thermal APPs. The relation between the electron temperature $\left(T_{e}\right)$ and $T_{e x c}$ can be also derived through collisional-radiative modeling of argon lines. Here, we use the method for the electron temperature versus excitation temperature reported for a range of plasma parameters (electron density and gas temperature) that covers measured values for our plasma. ${ }^{27} \mathrm{We}$ therefore estimate $T_{e}$ for our plasma using the measured $T_{e x c}$ and the method reported previously. ${ }^{27}$ Using the methodologies and procedures described above, we have measured gas temperature, electron density, and the electron temperature, which resulted to be $T_{g}=490 \mathrm{~K}, n_{e}=1.8 \times 10^{20} \mathrm{~m}^{-3}$, and $T_{e}=1.1 \mathrm{eV}$, respectively.

In our model, a non-thermal APP with noble gases (e.g., argon) is considered. The effect of hydrogen atoms on heating of NPs has been discussed in low-pressure silane plas$\operatorname{mas}^{18,21}$ and is neglected here. The particle temperature is obtained from the balance of energy fluxes to and from the surface of a particle assuming a stationary state. The recombination of ions with electron and the transferred kinetic energy of ions and electrons on the surface of the particle are the heating source while conduction through gas is the only non-negligible cooling term. In the first step, the particle potential/charge is calculated and it will be used for calculating the flux of charged particles.

The orbital motion limited (OML) model has been widely used in the literature for calculating the electric potential (charge) of a particle immersed in low pressure plasmas. ${ }^{28}$ Through the OML model, the particle potential is assumed equal to the floating potential that is calculated from equalizing electron and ion fluxes to the particle surface, i.e., $I_{e}=I_{i}$. Following the OML formulation, $I_{e}$ is obtained from ${ }^{29,30}$

$$
I_{e}=\pi a^{2} n_{e}\left\langle v_{e}\right\rangle \exp \left(\frac{e V_{p}}{k_{b} T_{e}}\right),
$$

where $a, n_{e}$, and $k_{b}$ are the particle radius, electron density, and Boltzmann constant, respectively, and

$$
\left\langle v_{e}\right\rangle=\left(\frac{8 k_{b} T_{e}}{\pi m_{e}}\right)^{1 / 2}
$$

is the thermal velocity of electrons averaged over the Maxwellian velocity distribution function; $V_{p}$ is the not-yet determined floating potential.

The ion current in the OML model is derived assuming collision-less motion of ions through

$$
I_{O M L}=\pi a^{2} \sqrt{\frac{8 k_{b} T_{i}}{\pi M}} n_{i}\left[1-\frac{e V_{P}}{k_{b} T_{i}}\right],
$$

where $n_{i}, M$, and $T_{i}$ are ion density, mass, and temperature, respectively, while $n_{i}=n_{e}$ due to charge neutrality, and it is also assumed that $T_{i}=T_{g}$. The OML model gives acceptable values for the floating potential under the condition $l_{i} \gg \lambda \gg a$, where $l_{i}$ is the ion mean free path and $\lambda$ is the plasma screening length.

However, a number of works reported in the literature have argued that the OML can overestimate particle charge/ potential at higher levels of ion collisonality. ${ }^{31-33}$ The effect of ion collisions and particularly charge-exchange collisions with neutrals plays an important role, as it can lead to the formation of trapped ions in a potential well around the particle. The analytical expression

$$
I_{i}=\left[1 / I_{i}^{W C}+1 / I_{i}^{S C}\right]^{-1}
$$

describes the ion current to the particle surface taking in consideration the effect of ion-neutral collisions, i.e., the collision-corrected model (CCM). ${ }^{33-36}$

In Eq. (4), $I_{i}^{W C}$ is the current of ions in a weakly collisional (WC) regime

$$
I_{i}^{W C} \approx \pi a^{2} n_{i} \sqrt{\frac{8 k_{b} T_{i}}{\pi M}}\left[1-\frac{e V_{P}}{k T_{i}}+0.1\left(-\frac{e V_{P}}{k_{b} T_{i}}\right)^{2}\left(\frac{\lambda}{l_{i}}\right)\right],
$$

i.e., with one or no collisions within the capture radius around the particle.

Equation (5) reduces to (3) in the limit of $l_{i} \rightarrow \infty$. The term

$$
I_{i}^{\mathrm{SC}}=4 \pi a n_{i} l_{i} v_{T_{i}} \frac{e\left|V_{p}\right|}{k_{b} T_{i}}
$$

describes the contribution of ions in a strongly collisional (SC) regime where more than one collision is expected.

For our model, we will use Eq. (4) to evaluate the ion current which provides accurate values for the ion current in the collisionless and weakly collisional regime, and it is an appropriate interpolation for both the weakly and strongly collisional regimes. For the range of the plasma parameters of this work, the collisionality lies in the weakly collisional regime, and $I_{i}$ approaches $I_{i}^{W C}$.

For evaluating the NPs heating mechanisms, we consider a noble gas atmospheric-pressure plasma with singly charged positive ions. For a small particle (diameter $<100 \mathrm{~nm}$ ), we write the steady-state energy balance ${ }^{21}$ on the surface of the particle as

$$
q_{\text {con }}=q_{\text {kin }}+q_{\text {rec }},
$$

where $q_{k i n}$ and $q_{\text {rec }}$ represent the rates of energy transfer via kinetic energy of charged species (ions and electrons) and ion-electron recombination on the particle surface, while $q_{c o n}$ is the heat loss rate to the surrounding gas through heat conduction.

The regime of heat conduction depends on the ratio of the mean free path of the gas molecules to the particle radius, determined by the Knudsen number $K n=l / a$. In the limit of large Knudsen numbers (typically $K n>10$ ), the heat conduction is in the free molecular regime while in the limit of very small Knudsen numbers $(K n<0.01)$, a continuum regime can be assumed for evaluating the heat conduction. Several 
approaches have been demonstrated for evaluating the heat loss rate in the transition regime, i.e., $K n \sim 1$.

The following relation: ${ }^{37}$

$$
q_{\text {con }}=\frac{2 \pi(2 a)^{2} K}{(2 a)+l G}\left(T_{p}-T_{g}\right),
$$

has been widely used in the literature for both the molecular regime as well as the transition regime. In Eq. (8)

$$
G=\frac{8 f}{\alpha(\gamma+1)} \quad \text { and } \quad f=(9-\gamma) / 4,
$$

where $K$ and $\gamma$ are the thermal conductivity and the specific heat ratio of the gas, and $\alpha$ is the thermal accommodation coefficient. We assume full accommodation $\alpha=1$ that is not far from reality. ${ }^{38}$ The mean free path is

$$
l=\frac{K}{p f}(\gamma-1)\left(\frac{\pi M T_{g}}{2 k_{b}}\right)^{0.5},
$$

as defined by McCoy and Cha. ${ }^{37}$

For a particle radius in the range of $1-100 \mathrm{~nm}$, and for $l \approx 81 \mathrm{~nm}$ (argon at atmospheric pressure and at $400 \mathrm{~K}$ ), the Knudsen number is in the range $0.8<K n<81$; therefore, Eq. (8) will be used in our model.

In Eq. (7)

$$
q_{k i n}=I_{i}\left(2 k_{b} T_{e}-e V_{p}\right)
$$

is calculated from the average kinetic energy of the ions/electrons reaching the particle, given by $-e V_{p}$ for the ions and by $2 k_{b} T_{e}$ for the electrons.

The term $q_{\text {rec }}=I_{i} \varepsilon_{i}$ in Eq. (7) is the energy released due to the recombination of impinging ions with (free) electrons on the particle surface (for $\operatorname{Ar} \varepsilon_{i}=15.76 \mathrm{eV}$ ).

In Fig. 3, the results of the NP potential and the ratio of the $I_{i} / I_{O M L}$ calculated for the plasma conditions described are presented. The potential is calculated in the CCM using Eq. (4) for the ion current which takes into account the effect of ion-neutral collisions; the potential from OML, using

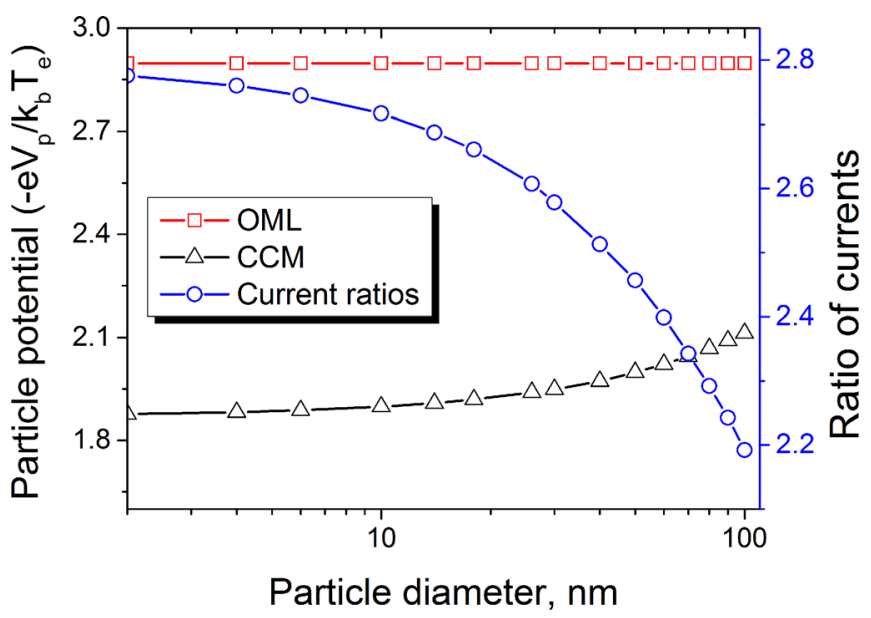

FIG. 3. The influence of collision-enhanced current on the nanoparticle potential for particles with diameter in the range of $2-100 \mathrm{~nm}$; the potential reported has been calculated with the OML model and with the CCM. The ratio of the corresponding currents $I_{C C M} / I_{O M L}$ is also presented.
Eq. (3), is also shown for the comparison. The potential obtained from CCM is lower than the OML value, as expected, and increases with increasing of the particle diameter. The ratio of CCM to OML ion currents decreases from 2.78 at $2 \mathrm{~nm}$ particle diameter to 2.19 for $100 \mathrm{~nm}$ particle diameter.

This model allows us to calculate the NP temperature from the energy balance Eqs. (7)-(11). Figure 4 shows the particle temperature calculated using the OML and CCM approximations. The calculated temperature from the OML model is $T_{p}=602 \mathrm{~K}$ for a $2 \mathrm{~nm}$ particle and increases to $619 \mathrm{~K}$ for a $100 \mathrm{~nm}$ particle. From the CCM model, $T_{p}=786 \mathrm{~K}$ ( $2 \mathrm{~nm}$ diameter particle $)$ and it shows a decreasing dependence on the increasing particle size. The difference between $T_{p}$ from CCM and OML for a $2 \mathrm{~nm}$ particles is $184 \mathrm{~K}$ representing a considerable effect of collisionenhanced ion current on the particle temperature.

For silicon NPs crystallization temperatures of $773 \mathrm{~K}$, $1073 \mathrm{~K}, 1173 \mathrm{~K}$, and $1273 \mathrm{~K}$ have been reported for NPs with diameters of $4 \mathrm{~nm}, 6 \mathrm{~nm}, 8 \mathrm{~nm}$, and $10 \mathrm{~nm}$, respectively, ${ }^{39}$ and suggest a temperature below $773 \mathrm{~K}$ would be required for NPs of the size reported here. The model shows that NPs below $4 \mathrm{~nm}$ can easily reach the reported crystallization temperatures ( $>780 \mathrm{~K}$, Figure 4 ) confirming effective particle heating in APPs as observed in our experiments.

In summary, we have demonstrated that the crystalline silicon nanoparticles can be nucleated and grown in atmospheric-pressure low-temperature microplasmas at gas temperatures well below the Si crystallization threshold. This phenomenon was explained by the efficient heating of the nanoparticles in non-thermal atmospheric pressure plasmas. The analysis of the energy balance on the surface of particles shows that effective heating of NPs can occur also in atmospheric pressure plasmas and if the ion density is sufficiently high. The comparison between the OML and CCM models reveals important differences as the energy flux supplied by the ion current to the NPs is enhanced in a collisional regime, leading to higher NP temperatures. The model allows for estimating particle temperature from the plasma parameters, providing very useful information for the design of plasma reactor/microreactor. These results have been used for the design of our high-throughput scalable NP synthesis reactor

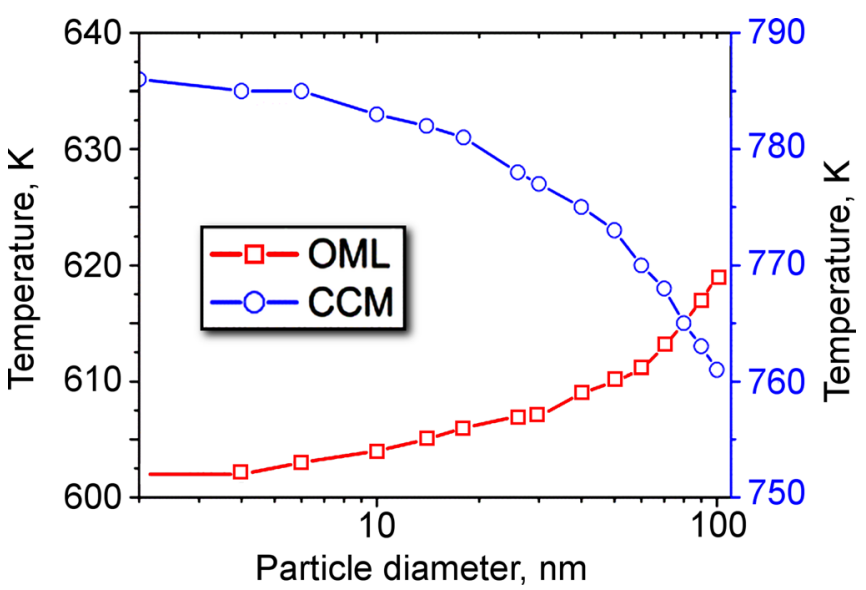

FIG. 4. Particle temperature for a range of nanoparticle diameter according to the two theoretical frameworks, OML, and CCM. 
which will be reported in the near future. Importantly, while we have exemplified our analysis for Si NPs, this work is applicable to a wide range of NPs and therefore of interest to nanotechnologists and nanoscientists working with different materials.

This work was supported by the Royal Society International Exchange Scheme (IE120884), the Leverhulme International Network (IN-2012-136), and EPSRC (EP/K022237/1). S.A. thanks the financial support of the University of Ulster Vice-Chancellor Studentship. I.L. and K.O. acknowledge financial support from CSIRO and Australian Research Council.

${ }^{1}$ E. D. Sargent, Nat. Photonics 6, 133 (2012).

${ }^{2}$ Y. Shirasaki, G. J. Supran, M. G. Bawendi, and V. Bulović, Nat. Photonics 7, 13 (2013).

${ }^{3}$ J. Fan and P K. Chu, Small 6, 2080 (2010).

${ }^{4}$ D. K. Kim, Y. Lai, B. T. Diroll, C. B. Murray, and C. R. Kagan, Nature Commun. 3, 1216 (2012).

${ }^{5}$ D. Mariotti, S. Mitra, and V. Švrček, Nanoscale 5, 1385 (2013).

${ }^{6}$ V. Švrček, M. Kondo, K. Kalia, and D. Mariotti, Chem. Phys. Lett. 478, 224 (2009).

${ }^{7}$ V. Švrček, D. Mariotti, and M. Kondo, Appl. Phys. Lett. 97, 161502 (2010).

${ }^{8}$ K. Ostrikov, E. C. Neyts, and M. Meyyappan, Adv. Phys. 62, 113 (2013).

${ }^{9}$ D. Mariotti and K. Ostrikov, J. Phys. D: Appl. Phys. 42, 092002 (2009).

${ }^{10}$ D. Mariotti and R. M. Sankaran, J. Phys. D: Appl. Phys. 43, 323001 (2010).

${ }^{11}$ T. Belmonte, G. Arnoult, G. Henrion, and T. Gries, J. Phys. D: Appl. Phys. 44, 363001 (2011).

${ }^{12}$ W.-H. Chiang, C. Richmonds, and R. M. Sankaran, Plasma Sources Sci. Technol. 19, 034011 (2010).

${ }^{13}$ R. M. Sankaran, D. Holunga, R. C. Flagan, and K. P. Giapis, Nano Lett. 5, 537 (2005).

${ }^{14}$ T. Nozaki, K. Sasaki, T. Ogino, D. Asahi, and K. Okazaki, Nanotechnology 18, 235603 (2007).

${ }^{15}$ S. Q. Xiao and S. Xu, J. Phys. D: Appl. Phys. 44, 174033 (2011).
${ }^{16}$ F. Priolo, T. Gregorkiewicz, M. Galli, and T. F. Krauss, Nat. Nanotechnol. 9, 19 (2014)

${ }^{17}$ A. J. Nozik, Nano Lett. 10, 2735 (2010).

${ }^{18}$ L. Mangolini and U. Kortshagen, Phys. Rev. E 79, 026405 (2009).

${ }^{19}$ J. E. Daugherty and D. B. Graves, J. Vac. Sci. Technol., A 11, 1126 (1993).

${ }^{20}$ G. H. P. M. Swinkels, H. Kersten, H. Deutsch, and G. M. W. Kroesen, J. Appl. Phys. 88, 1747 (2000).

${ }^{21}$ H. R. Maurer and H. Kersten, J. Phys. D: Appl. Phys. 44, 174029 (2011).

${ }^{22}$ S. Hofmann, A. F. H. van Gessel, T. Verreycken, and P. Bruggeman, Plasma Sources Sci. Technol. 20, 065010 (2011).

${ }^{23}$ S. Y. Moon and W. Choe, Spectrochim. Acta, Part B 58, 249 (2003).

${ }^{24}$ J. Luque and D. R. Crosley, SRI International Report No. MP-99-009, 1999.

${ }^{25}$ S. G. Belostotskiy, T. Ouk, V. M. Donnelly, D. J. Economou, and N. Sadeghi, J. Appl. Phys. 107, 053305 (2010).

${ }^{26} \mathrm{~S}$. Namba, T. Yamasaki, Y. Hane, D. Fukuhara, K. Kozue, and K. Takiyama, J. Appl. Phys. 110, 073307 (2011).

${ }^{27}$ X.-M. Zhu, W.-C. Chen, and Y.-K. Pu, J. Phys. D: Appl. Phys. 41, 105212 (2008).

${ }^{28}$ J. E. Allen, Phys. Scr. 45, 497 (1992).

${ }^{29}$ S. V. Vladimirov, K. N. Ostrikov, M. Y. Yu, and L. Stenflo, Phys. Rev. E $\mathbf{5 8}, 8046$ (1998).

${ }^{30}$ M. Keidar, O. R. Monteiro, A. Anders, and I. D. Boyd, Appl. Phys. Lett. 81, 1183 (2002).

${ }^{31}$ M. Lampe, V. Gavrishchaka, G. Ganguli, and G. Joyce, Phys. Rev. Lett. 86, $5278(2001)$

${ }^{32}$ A. V. Zobnin, A. P. Nefedov, V. A. Sinelshchikov, and V. E. Fortov, J. Exp. Theor. Phys. 91, 483 (2000).

${ }^{33}$ S. A. Khrapak, S. V. Ratynskaia, A. V. Zobnin, A. D. Usachev, V. V. Yaroshenko, M. H. Thoma, M. Kretschmer, H. Hofner, G. E. Morfill, O. F. Petrov, and V. E. Fortov, Phys. Rev. E 72, 016406 (2005).

${ }^{34} \mathrm{M}$. Gatti and U. Kortshagen, Phys. Rev. E 78, 046402 (2008).

${ }^{35}$ S. A. Khrapak and G. E. Morfill, Phys. Plasmas 15, 114503 (2008).

${ }^{36}$ L. G. D'yachkov, A. G. Khrapak, S. A. Khrapak, and G. E. Morfill, Phys. Plasmas 14, 042102 (2007).

${ }^{37}$ F. Liu, K. J. Daun, D. R. Snelling, and G. J. Smallwood, Appl. Phys. B 83, 355 (2006).

${ }^{38}$ W. M. Trott, J. N. Castañeda, J. R. Torczynski, M. A. Gallis, and D. J. Rader, Rev. Sci. Instrum. 82, 035120 (2011).

${ }^{39}$ M. Hirasawa, T. Orii, and T. Seto, Appl. Phys. Lett. 88, 093119 (2006).

${ }^{40}$ See supplementary material at http://dx.doi.org/10.1063/1.4872254 for further analysis of chemical and optical properties of Si nanoparticles. 